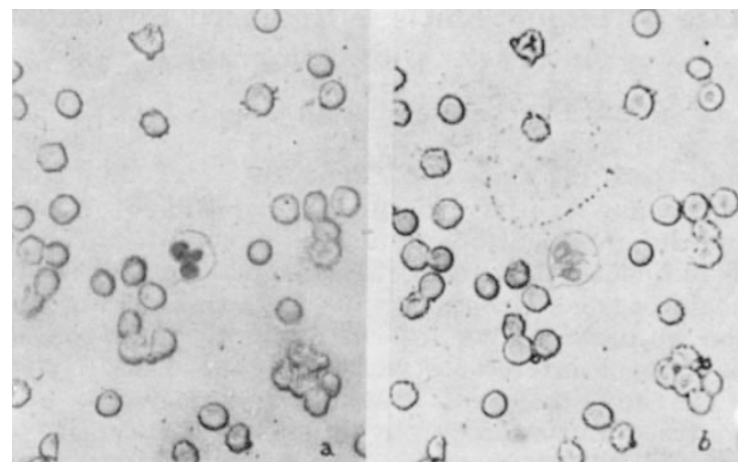

thickness. The whole is dried rapidly at room temperature by reducing the pressure to about $10 \mathrm{~cm}$. mercury ; this helps to force out air bubbles in the emulsion. After a suitable period of exposure the emulsion is processed in the usual way; but the plates have to be carefully washed, since traces of hypo increase the risk of peeling.

By using this method the cells appear to emerge from the treatment unharmed, and no distortion is observed either in the specimen or in the emulsion. Photomicrographs of a section obtained by this method, before and after coating with the $G .5$ emulsion, are reproduced herewith; the section contained blood cells labelled with radioactive zinc. Zinc-65 is not very suitable for track autoradiography ; but it was used in the study of a problem which will be described on another occasion. The erythrocytes and leucocyte, which are seen on the microphotograph together with an electron track $(b)$, appear exactly the same as on the original section $(a)$.

This work has been carried out with the aid of a grant from the Medical Research Council, to which we express our indebtedness.

Mary BLUNDELI

J. ROTBLAT

Physics Department,

Medical College of

St. Bartholomew's Hospital, London, E.C.1.

' King, D. T., Harris, J. E., and Tkaczyk, S., Nature, 187, 273 (1951). ${ }^{2}$ Boyd, G. A., and Levi, H., Science, 111, 58 (1950).

${ }^{3}$ Harris, J. E., Sloane, J. F., and King, D. T., Nature, 166, 25 (1950). 4 Campbell, D., Nature, 167, 274 (1951).

s Boyd, G. A., and Board, F. A., Science, 110, 586 (1949).

- Pelc, S. R., Nature, 160, 749 (1947).

Belanger, L. F., and Leblond, C. P., Endocrin., 39, 8 (1946).

\section{Excitation of Nitrogen in a Sodium Arc}

SPECTROGRAMS have been obtained of a high current-density sodium arc obtained with a lowdispersion Hilger quartz spectrograph, in the region of 4000-2000 A. The cathode was a sodium pool in a 'Pyrex' glass tube of about $2 \mathrm{~cm}$. diameter, and the anode was a hollow nickel-plated iron rod cooled by a stream of oil. The voltage supply for the highcurrent arc (2-6 amp.) was obtained from a 120-volt storage battery, and the low pressure inside the tube was maintained by a 'Cenco Hyvac' pump.

The first negative bands of nitrogen are brought out prominently along with the principal series lines of sodium at the higher current strengths used. Herman $^{1}$ found an increase in the intensity of the first negative system, after the break-up of a glow discharge in nitrogen, and ascribed it to the recom- bination of $\mathrm{N}_{3}++$ ions with electrons. In the sodium arc containing an admixture of nitrogen, the excitation of nitrogen, presumably, arises from the collisions of the first kind between electrons and ground-state $X^{\prime} \Sigma N_{2}$ molecules ${ }^{2}$.

\author{
First Grade Colloge, \\ Mysore. \\ Nov. 28. \\ 1 Herman, L., and Herman, R., Nature, 161, 1018 (1948). \\ 2 Nicholls, R. W., Nature, 162, 231 (1948).
}

C. K. Sundarachar

\section{De Haas - van Alphen Effect in Aluminium and Antimony}

THE de Haas - van Alphen effect (periodic field dependence of magnetic susceptibility) has been found in aluminium and antimony single crystals at $4.2^{\circ} \mathrm{K}$. and lower temperatures. The effect depends in a complicated way on the direction of the mag. netic field, and there are complicated modulation effects similar to those previously found in other metals $^{1}$. Typical values of the period at 15,000 gauss are 400 gauss in aluminium and 270 gauss in antimony; the amplitude in both metals increases by a factor of order 4 when the temperature is reduced from $4 \cdot 2^{\circ} \mathrm{K}$. to $1 \cdot 5^{\circ} \mathrm{K}$. Aluminium is the first metal of cubic symmetry in which the effect has been found.

\section{SHOENBERG}

Royal Society Mond Laboratory, Cambridge. March 10.

Dingle, R. B., and Shoenberg, D., Nature, 168, 652 (1950).

\section{An Examination of Weld Metal by means of the Electron Microscope}

DURING a recent investigation into the cause of variation in the temperature of transition from rough to brittle behaviour of weld metal, the X-ray powder diffraction patterns of the non-metallic inclusions separated from different samples gave evidence of the presence of crystalline rhodonite (a manganese silicate belonging to the triclinic system) in weld metal of low transition temperature only, whereas chemical analysis indicated the same elemental composition of the inclusions in all cases.

Examination by means of the optical microscope failed to confirm this observation, and as a result two samples of weld metal having high and low transition-temperatures were sent to this Laboratory for electron microscopic examination.

Negative 'Formvar' replicas, shadowed with chromium at an angle of 1 to 4, were made in the normal manner after the metals had been polished and etched. The etching consisted of a ten-minute treatment with a cold 5 per cent solution of hydrochloric acid (sp. gr. 1.18 aqueous) in ethanol, in which rhodonite is known to be soluble, followed by a light treatment with 2 per cent 'Nital' to bring out the normal microstructure. Several replicas were taken off each sample to ensure that any observed differences were truly representative.

Replicas taken from the low-transition-temperature metal revealed numerous well-defined pits, of which those seen in Figs. 1 and $2(\times c .8,600)$ are typical. 